\title{
El cuidado enfermero como problema ético: concepto y principios prácticos aplicados al acto de cuidado
}

O cuidado de enfermagem como problema ético: conceitos e princípios aplicados ao ato de cuidar Nursing care as an ethical problem: concepts and principles applied to the act of caring

\author{
Alejandro Miranda', Sebastián Contreras' \\ ' Universidad de los Andes, Facultad de Derecho. Santiago, Chile.
}

Submissão: 25-07-2013 Aprovação: 16-10-2014

\section{RESUMEN}

En este trabajo los autores estudian la naturaleza del acto del cuidado, destacan la importancia de la ética en las profesiones vinculadas con la salud de las personas y desarrollan, a la luz de la tradición central de la filosofía moral occidental, un conjunto de principios que deben guiar la actividad de la enfermería.

Palabras clave: Ética en Enfermería; Atención de Enfermería; Enfermería; Atención Dirigida al Paciente.

\section{RESUMO}

Neste trabalho, os autores refletem sobre a natureza do ato de cuidar, enfatizam a importância da ética nas profissões relacionadas com a saúde das pessoas e desenvolvem, à luz da tradição central da filosofia moral ocidental, um conjunto de princípios que deve orientar o trabalho de enfermagem.

Descritores: Ética em Enfermagem; Cuidados de Enfermagem; Enfermagem; Assistência Centrada no Paciente.

\section{ABSTRACT}

In this paper the authors study the nature of the act of care, emphasize the importance of ethics in the professions related to the health of people and develop, in the light of the central tradition of Western moral philosophy, a set of principles that should guide nursing activity.

Key words: Ethics Nursing; Nursing Care; Nursing; Patient-Centered Care. 


\section{PLANTEAMIENTO}

El fenómeno del "cuidado" ha existido desde siempre. Originalmente asociado al problema del mantenimiento de la vida, hoy se alza como una actividad profesional que combina los conocimientos de la técnica con principios de prudencia derivados del precepto fundamental de respecto por la vida y dignidad de la persona, para así conseguir una adecuada planificación, organización y control de la provisión de los tratamientos más oportunos, seguros y eficaces para la recuperación del enfermo.

Pese a sus fundamentos "naturales", el cuidado profesional no se identifica con el cuidado humano en general. La Enfermería es una ciencia y una disciplina autónoma dentro de las profesiones de la salud. Implica un ideal de protección y promoción del bienestar humano que pone el acento en la unidad esencial de cuerpo y mente ${ }^{(1)}$, haciendo hincapié en que los problemas físicos son también afecciones espirituales y que los estados del ánimo pueden convertirse en problemas de salud.

El propósito de la Enfermería es decidir el mejor curso de acción para la recuperación del enfermo, en el momento indicado, en el lugar que debe ser y con las personas indicadas. En este sentido, el ejercicio de la Enfermería tiene las dificultades propias de la praxis: en el mundo de la salud existe contingencia y cambio. Nunca una enfermedad es igual a la otra, porque los sujetos son todos distintos y tienen historias y experiencias diferentes.

Más que una actividad técnica, el cuidado representa una actitud profesional. Éste no se agota en el cumplimiento de tareas por parte del equipo médico. Es un ideal normativo que busca enaltecer la dignidad del enfermo como persona humana y miembro del cuerpo social. Como actitud profesional, pretende ir más allá de la dicotomía entre teoría y práctica, destacando que la recuperación de la salud es siempre un problema práctico-teórico: práctico, porque se deben decidir planes de acción para paliar una determinada enfermedad o dolencia; y teórico, porque esos cursos de acción no se pueden escoger sin un mínimo conocimiento de la realidad tal como es.

En este esfuerzo por dar una solución adecuada a la dolencia del enfermo, el profesional del cuidado debe conjugar aspectos técnicos con elementos históricos, emocionales y experienciales, incluso con cuestiones religiosas. Para esto no existe una pauta o receta magistral. El enfermero ha de aplicar sus conocimientos con la mayor diligencia posible, aspirando en todo caso a la promoción del bienestar integral de su paciente.

Dada la importancia de este asunto, y como una manera de contribuir a la reflexión sobre la moralidad de las decisiones enfermeras, hemos decidido ahondar en la naturaleza del acto de cuidado, haciendo hincapié en su carácter prudencial y artístico, así como en la necesidad de vincular la ética con las profesiones de la salud. Con el objeto de ser claros en nuestra exposición, dividiremos este trabajo de la siguiente manera: (i) discusión acerca de la importancia de la ética en el área de la salud; (ii) revisión de la naturaleza del acto de cuidado; (iii) presentación de algunos principios morales aplicables a la práctica enfermera; (iv) unas consideraciones finales.

\section{LA ÉTICA EN LAS PROFESIONES DE LA SALUD}

\section{¿Por qué es necesaria e importante la ética?}

La necesidad de la ética, no sólo en la Enfermería, sino en todas las actividades humanas, reside en el hecho de que los individuos están dotados de conocimiento intelectual y de libre voluntad. Su importancia, por otro lado, estriba en el hecho de que los hombres tienen un fin último que alcanzar, y no todas las acciones dirigen a él.

En virtud de su inteligencia, el hombre tiene la capacidad de conocer conceptos universales y abstractos. Esto es lo que lo distingue esencialmente de los animales inferiores. El conocimiento abstracto posibilita el desarrollo de las ciencias y las artes, y con ello la formación de una cultura. La consecuencia más importante de la posesión de inteligencia es la existencia del libre albedrío o libertad de elección: sólo los seres inteligentes son libres. Puesto que la inteligencia nos permite entender la noción general y abstracta del bien, podemos darnos cuenta de que los distintos bienes que se nos presentan no son bienes perfectos: tienen aspectos de bondad pero no son toda la bondad. En virtud de esta constatación, la voluntad puede dirigirse a ciertas opciones u objetos por lo que tienen de buenos, o puede no dirigirse a ellos por lo que les falta de bondad. Éste es, de modo muy simplificado, el argumento principal para demostrar la existencia del libre albedrío.

Los seres que no están dotados de inteligencia se mueven por instinto, y no tienen, por ello, ni la posibilidad ni la necesidad de elegir (en sentido relevante). Todos reconocemos esto, y por eso no llevamos a un tribunal, acusándolo de homicidio, al león que devora al guardaparques. El león, por no gozar de libre albedrío, está fuera del orden moral. Los seres humanos, en cambio, por su libertad, se constituyen como agentes morales. Un corolario de esto es que los seres humanos son responsables por sus acciones: puesto que ha estado en su poder realizar o no ciertas acciones, los hombres están en posición de responder por ellas. Y aquí ya estamos en presencia del fenómeno de lo moral.

Por otra parte, por la experiencia interna, los hombres pueden constatar que sus acciones se estructuran teleológicamente, es decir, se dirigen a alcanzar ciertos fines. Como no es posible que todos fin sea sólo un medio para alcanzar otro posterior (porque esto produciría una regresión al infinito que conduce al absurdo, los hombres pueden saber que ha de existir un fin último de la vida humana, o sea, algo bueno por sí mismo, que no se busque como medio para otra cosa. Desde muy antiguo, este bien ha recibido el nombre de felicidad - eudaimonía, la Ilamaban los griegos - , palabra que designa el logro de la perfección humana integral. Cuál sea el bien que objetivamente constituye esa felicidad es algo que ha dado lugar a muchas discusiones, y que no podríamos tratar en este lugar. Pero para destacar la importancia y la necesidad de la ética basta sólo con consignar el siguiente hecho: no todas las acciones que el hombre realiza lo dirigen hacia esa perfección integral. Es manifiesto que hay algunas que lo degradan, porque no permiten una actualización de sus potencialidades.

La ética es importante, en definitiva, porque es valioso conocer y poner por obra las acciones que nos dirigen a la perfección, 
y porque es valioso conocer, esta vez para omitir, las acciones que impiden u obstaculizan nuestra realización integral.

\section{La ética en las profesiones de la salud}

La razón humana tiene una aptitud natural para conocer los principios supremos de la moralidad. A partir de estos principios, es posible deducir reglas morales más particulares, hasta alcanzar el nivel de las normas morales específicas, es decir, de las normas que mandan o prohíben tipos concretos de acciones humanas (por ejemplo, la norma que prohíbe el homicidio o la que ordena cumplir las promesas). Las normas morales específicas se van especializando cada vez más, en forma directamente proporcional a la especialización de los distintos campos de acción. Así, surgen normas morales específicas relativas a la actividad propia de los abogados y jueces, de los periodistas o de los profesionales de la salud.

Estas normas no son algo completamente nuevo: más bien son una particularización de principios más generales, que se aplican ahora a los problemas de una determinada disciplina, ciencia o arte. Discernir si es lícito buscar la absolución de un acusado que se sabe que es culpable, es algo que interesa principalmente a los abogados; saber si es lícito hacer grabaciones con cámaras ocultas es algo que interesa principalmente a los periodistas; y saber si es lícito hacer una transfusión sanguínea forzada a un paciente Testigo de Jehová, cuando no se puede salvar su vida de otro modo, es algo que interesa principalmente a los profesionales de la salud. Desde luego, estas cuestiones interesan de algún modo a todos los miembros de la comunidad, pero son especialmente apremiantes para quienes tendrán que tomar inmediatamente las decisiones que estos problemas morales demandan.

La ética profesional o deontología profesional es, por consiguiente, la parte de la ética especial que tiene por objeto solucionar los problemas morales que surgen en el contexto propio de una determinada ocupación humana. Este nivel de especialización se hace necesario a lo menos por tres razones. En primer lugar, porque las normas morales relativas a una cierta actividad responden en gran medida a la comprensión que se tenga de los fines de esta actividad, y los fines de una cierta actividad se comprenden mejor desde dentro de ella, es decir, los comprenden más adecuadamente quienes la ejercen. (Piénsese, por ejemplo, en el documento Los fines de la medicina, elaborado por el The Hastings Center, que ha sido de gran ayuda para profundizar en el conocimiento de las obligaciones de los que se dedican a las profesiones asistenciales).

El nivel de especialización propio de la ética profesional es necesario, en segundo lugar, porque las circunstancias que rodean los contextos de acción propios de ciertos saberes y oficios pueden alejarse mucho de las circunstancias de los casos comunes. Y la especialización es necesaria, finalmente, porque la adecuada selección de los principios morales exige un conocimiento cabal de los hechos que pretenden ser valorados o descritos en términos morales. Si no se sabe, por ejemplo, cómo se realiza una salpingectomía para tratar un embarazo ectópico, no se puede tener un juicio claro sobre su moralidad.

\section{LA NATURALEZA DEL ACTO DE CUIDADO}

El ideal moderno de la matematización de la ciencia no tiene cabida en el ejercicio de la Enfermería. Los actos de cuidado no se originan en deliberaciones mecánicas o automatizadas del profesional. Son siempre actuaciones prudenciales, circunstanciadas, enmarcadas en unas coordenadas de tiempo y lugar muy precisas y cambiantes. Por ende, están "teñidos" de aspiraciones, motivaciones, cultura, etc., y no pueden ser presentados como meras reacciones instintivas (o computarizadas) del enfermero ante una dolencia o patología determinada.

Ante la pregunta por la naturaleza del acto de enfermería alguien podría pensar que el cuidado profesional es un asunto sencillo, y que no es algo distinto del cuidado "natural" que una madre ejerce con sus hijos. Nada más alejado de la realidad. El trabajo profesional del enfermero constituye un proceso complejo asociado a las distintas etapas de la vida. Desde el punto de vista técnico, equivale a un conjunto de procesos que buscan "prescribir cursos de acción para el fomento, prevención y recuperación de la salud, a partir del conocimiento de enfermería y de las ciencias que ayudan a comprender la salud"(2).

El cuidado enfermero representa un juicio clínico sobre la respuesta de un individuo a sus problemas de salud, reales o potenciales. A causa de ese juicio, como señala NANDA-I 2008, el enfermero será responsable de la monitorización de las respuestas del paciente, de la adopción de decisiones que culminarán en un plan de cuidados y de la ejecución de los cursos de acción resueltos como los más pertinentes, incluyendo la colaboración interdisciplinar y la derivación del paciente a otras unidades, si fuera necesario.

Lo anterior es una muestra de que el acto de cuidado supone un trabajo sumamente complicado, "ya que incluye la posesión de conocimientos técnicos, una gran cantidad de conocimientos formales, capacidad de comunicación, complicidad emocional y otras muchas cualidades"(3). Como acto profesional, se funda en lo que la doctrina jurídico-moral ha denominado obligación de medios, y se especifica por la concurrencia de tres notas esenciales: la ejecución típica (por la que el enfermero se ciñe a una determinada lex artis), el principio de búsqueda de recuperación del enfermo, y la licitud (moral y legal).

Tales propiedades del acto de enfermería no son otra cosa, creemos, que una manifestación del carácter profesional de ese acto de cuidado. Como se ha dicho, las decisiones enfermeras no son una simple extensión del cuidado humano en general al campo de las ciencias médicas. Ciertamente hunden sus raíces en las prácticas de cuidado "natural" que todas las culturas han mostrado, pero no se pueden presentar como puras aplicaciones de ese fenómeno a casos complejos o en donde exista riesgo vital.

En efecto, si bien todas las disciplinas tienen su punto de partida en un comportamiento humano "natural", como el cuidado, la enseñanza, la construcción, la estrategia, etc., esas conductas "naturales" no son idénticas a las actividades profesionales que se originan en ellas. Los abogados, por ejemplo, aunque ponen en ejercicio una actitud "natural" de defensa y 
promueven un también "natural" espíritu de justicia, realizan una tarea social que no se reduce a esas "actitudes naturales". En las profesiones existe una obligación específica de cumplimiento del encargo del cliente. Los profesionales están en una posición de garante que asumen voluntariamente. De esa relación entre el profesional y quien requiere sus servicios surgen deberes y derechos recíprocos que no se encuentran en la conducta "natural" que dio origen a esa actividad: la madre que enseña a su hijo los nombres de los planetas del Sistema Solar, no tiene por qué saber la distancia entre esos planetas y la Tierra. El profesor que esa madre ha contratado para explicar a su hijo nociones de astronomía sí debe saberlo.

Entonces, a diferencia del cuidado "natural", el cuidado "enfermero" está sometido a ciertas reglas o criterios técnicos. En primer lugar, el Ilamado acto profesional de cuidado debe sujetarse al principio de normalidad. Aplicado al campo de la salud, la normalidad dice relación con criterios de frecuencia estadística, "en cuanto a características físicas y funcionales de todos los individuos pertenecientes al grupo etario y racial del paciente en cuestión"(4). Lo normal es aquello que, caeteris paribus, ocurre la mayoría de las veces. Es normal, por ejemplo, que en un proceso infeccioso el paciente tenga ciertos niveles de fiebre y malestar general. Pero la fiebre y el malestar físico son sólo indicadores. Por sí mismos, son insuficientes para la realización de un diagnóstico de enfermería y para tomar una decisión definitiva sobre un cierto tipo de cuidado. Podría suceder que el sujeto no presente ni fiebre ni dolor muscular y aun así esté desarrollando un proceso infeccioso. O podría pasar, a la inversa, que el paciente tenga episodios de fiebre y malestar, pero que esto no se deba a una infección, sino a una insolación o a una alergia (patología que, habitualmente, no está asociada a la fiebre).

La pura normalidad estadística no es un criterio suficiente para elegir un cierto tratamiento médico. La matemática no logra registrar todos los casos posibles; sólo pretende tener un control de los casos "normales". Este límite del conocimiento técnico debe ser superado prudencialmente. El enfermero, que sabe que cada enfermedad es distinta (y que cada enfermo es también diferente), deberá combinar los datos extraídos de la entrevista con el paciente con otros conocimientos propios de su experiencia. Sólo así podrá tomar la decisión más adecuada para una dolencia o enfermedad.

Elegido un curso de acción, el profesional del cuidado deberá procurar el restablecimiento de la funcionalidad del paciente, aunque no de la funcionalidad absoluta sino solamente de su funcionalidad operativa. Es decir, el cuidado enfermero no busca recuperar completamente al sujeto. Esto sería una pretensión absurda. Muchas veces es imposible que el paciente vuelva a su anterior estado de salud. La gestión del cuidado, por ende, no aspira a que el sujeto se restablezca por completo. Aspira, y no debe ser de otro modo, a que el paciente logre reincorporarse a la dinámica social. Esto es verdaderamente interesante. El tratamiento personal con el enfermo podría ocasionar que el profesional del cuidado desarrolle trastornos ansiosos o frustraciones por su imposibilidad para retrotraer la situación del sujeto hasta antes de la patología. Es fundamental, por este motivo, que el enfermero conozca sus limitaciones personales, así como las limitaciones de su arte y de la enfermedad que atiende a la hora de Ilevar a cabo a la gestión del cuidado.

No es extraño que episodios traumáticos asociados al no restablecimiento del enfermo se presenten en los profesionales de la Enfermería. Por la propia naturaleza de sus funciones, el enfermero es el miembro del equipo médico que mantiene una relación más cercana con el paciente. Una forma de “ligazón" une al enfermo y al profesional en la práctica del cuidado. De ahí que se haya escrito que la relación de mutua confianza y complicidad entre profesional y paciente es un elemento esencial del acto de enfermería. No hay gestión del cuidado sin dedicación y empatía.

Puesto que la Enfermería es una actividad que presupone un cierto ideal de excelencia humana, además de estar sometida al principio de búsqueda de recuperación del enfermo y a una determinada lex artis propia de su actividad profesional, está también condicionada por el deber de actuación lícita, tanto en el plano de la Ética como en el campo del Derecho. Surge así el criterio técnico de la actuación según ciencia y conciencia. Los enfermeros, cuando toman decisiones, no ejecutan un plan de trabajo rígido y máximamente detallado. No existe un manual que señale lo que se debe hacer en cada caso. Sólo hay principios generales y criterios de orientación. Por este motivo, cada decisión de enfermería es completamente diferente y engloba ciertos riesgos y dificultades. A veces, un determinado tratamiento provoca efectos no deseados. Podría darse el caso de que un medicamento para el dolor de cabeza cause malestares estomacales aun más difíciles de controlar que la cefalea.

Como una medida de prudencia ante las contingencias que envuelven a la práctica de los enfermeros, se ha establecido que el cuidado, para ser bueno desde el plano técnico y moral, debe ser un fenómeno simultáneo. Cuando los teoristas de la Enfermería hablan de la simultaneidad del cuidado, intentan poner de relieve que la actividad enfermera debe procurar la interacción no sólo del paciente y su tratante, sino también de sus familiares y de los otros profesionales del equipo de salud ${ }^{(5)}$.

Todo lo expuesto muestra la alta complejidad de los cuidados enfermeros. Las decisiones de los profesionales del cuidado están siempre condicionadas por factores cambiantes como la edad, el sexo y la existencia de alguna patología de base en el paciente. No existe, prima facie, una solución correcta para cada enfermedad o dolencia. Pese a esto, los enfermeros siempre deben tomar la decisión más adecuada. Como una manera de ayudar a la reflexión sobre este problema, a saber, sobre las dificultades que envuelven a todo acto de enfermería, a continuación desarrollamos algunos principios éticos que, en nuestra opinión, podrían aplicarse directamente a la gestión del cuidado.

\section{PRINCIPIOS FUNDAMENTALES DE LA ÉTICA DEL CUIDADO ENFERMERO}

Sin intención de ser exhaustivos, y como una herramienta de apoyo a los profesionales de la Enfermería, proponemos a continuación cuatro principios o reglas prácticas que 
debieran regir la actividad profesional del cuidado. No se trata de los únicos principios existentes. Sólo se trata de los que, en nuestra opinión, son los más relevantes.

\section{Primer principio: respeto por la dignidad de la persona}

La principal exigencia de justicia en el orden social es la defensa de la dignidad de la persona humana. Se utiliza el término "dignidad" para referirse a la excelencia por la que un ser destaca entre otros. En el sentido que aquí nos interesa, la palabra dignidad designa aquella excelencia o preeminencia que le corresponde a los seres que son personas, es decir, a los seres dotados de naturaleza racional. Puesto que todos los individuos de la especie humana son de naturaleza racional, todos los individuos de la especie humana son personas.

La naturaleza racional es lo que eleva a los individuos de la especie humana por sobre todos los demás seres del mundo visible. Lo característico de un ser personal es que, por estar naturalmente dotado de inteligencia y de libre voluntad, se constituye como un cierto fin en sí mismo. Filósofos que en algunos puntos difieren de manera importante, coinciden, sin embargo, en gran medida, en esta concepción de la dignidad de la persona humana.

Por ejemplo, Immanuel Kant, un filósofo de la modernidad, enseñaba que el más básico de los imperativos morales exige obrar de modo tal que uno se valga de la humanidad, tanto en su propia persona como en la persona de cualquier otro, siempre al mismo tiempo como fin, y nunca meramente como medio. Y Tomás de Aquino - un pensador de la cristiandad latina medieval- escribía que el hombre tiene dignidad en cuanto es naturalmente libre y existente para sí mismo, y añadía que un hombre, por su naturaleza, no se ordena a otro como a su fin.

La dignidad es el valor propio de los seres personales, mientras que el "precio" es el valor de las realidades que no son personas, esto es, de las que llamamos genéricamente "cosas". Las cosas no son fines en sí mismas: son medios o instrumentos que se ordenan al bien de las personas. Nunca, en cambio, una persona se ordena al bien de otra como mero medio. Eso equivaldría a negarle su valor intrínseco, a negarle la condición de fin en sí misma que le viene de su naturaleza racional.

La dignidad humana se viola, por consiguiente, cuando se trata a un ser humano como si fuera un mero medio o un instrumento, o sea, cuando se lo cosifica. La esclavitud es un ejemplo paradigmático de esta cosificación del hombre, pero no es el único: cuando se procura la muerte de un ser humano inocente como medio para cualquier fin, por muy noble que ese fin parezca, se está reduciendo a la víctima a la condición de mero medio. La tradición filosófica clásica concibe la existencia de unas normas morales de carácter absoluto - es decir, que no admiten excepción - que prohíben aquellos actos que son siempre y en sí mismos atentatorios contra la dignidad de la persona. Estos preceptos absolutos - que mandan abstenerse de realizar ciertos actos - constituyen los límites de la conducta humana. Si se transgreden esos límites, se degrada al hombre. Uno de los más importantes de estos preceptos absolutos es el que prohíbe toda destrucción directa o intencional de la vida humana inocente, que es lo que analizaremos en relación con el segundo principio.

\section{Segundo principio: se debe respetar la vida humana como un bien básico}

Este principio, si bien es importante en general, es fundamental en el caso de las profesiones vinculadas directamente con la salud y la vida de las personas. El respeto por la vida de los seres humanos inocentes desde el comienzo de su existencia hasta su muerte natural es una exigencia del reconocimiento de la dignidad de la persona humana. Esto es así porque negar el valor de la vida de una persona equivale a negar el valor de la persona como tal. En efecto, la vida no es propiamente algo que los vivientes tengan: el vivir es el mismo ser de los vivientes. Por lo tanto, si al viviente en cuestión le corresponde un tipo de ser valioso por sí mismo - como ocurre con el ser personal - , su vida ha de considerarse dotada de ese mismo valor.

La vida humana es, por consiguiente, intrínsecamente buena. Pero además es un bien de carácter básico, en el sentido de que es necesaria para la perfección humana integral: la perfección del viviente humano supone e incluye su vivir.

Desafortunadamente, las ideas anteriores no cuentan hoy con una aceptación unánime. En los últimos años se ha abierto paso una fuerte cultura antivida, que ve la vida humana como un bien de carácter meramente instrumental, y, por tanto, desechable cuando ya no es útil para conseguir un fin extrínseco a ella.

Así, hoy se suele afirmar que la vida biológica humana no es, en sí misma o intrínsecamente, un bien que merezca respeto. Quienes piensan de este modo arguyen que la vida biológica es sólo instrumentalmente buena, esto es, que sólo es buena en la medida en que estar vivo es una condición para tener un conjunto de experiencias que son intrínsecamente buenas. Cuando ya no se pueden tener estas experiencias, la vida deja de ser un bien.

Es indudable que esta concepción se ve favorecida por la mentalidad hedonista contemporánea, que cifra el fin de la existencia humana en el placer. Si la vida ya no permite tener experiencias placenteras, entonces esa vida ha dejado de ser útil, y, por lo mismo, ha dejado de ser un bien. Sin embargo, si se miran las cosas con atención, esta posición no se sostiene. Todos admiten, en efecto, que el fin último de una cosa es su realización plena. Ahora bien, como dice Patrick Lee,

la realización plena de una cosa no puede ser vista como valiosa y, a la vez, la cosa misma como de un valor meramente instrumental. La cosa misma no puede ser vista como instrumental a su bien - su bien debe incluir su ser además de la perfección de su ser ${ }^{(6)}$.

O sea, es lógicamente incoherente que alguien que busca su bien vea su completo ser como meramente instrumental para otro bien. El bien es la plenitud del ser, y la plenitud del ser ha de incluir, evidentemente, el ser.

Por lo tanto, la vida humana biológica sólo puede ser vista como un bien instrumental cuando la persona se identifica con la mera consciencia o con un sujeto no corpóreo. Esto es 
lo propio del dualismo. El dualismo es la posición filosófica que niega que los seres humanos sean esencialmente seres corpóreos. Según el dualismo, el cuerpo no forma parte de la persona humana: la persona únicamente se identifica con el sustrato de los actos intelectuales y volitivos. Por eso, cuando ya no hay actos intelectuales o volitivos, no hay persona.

Pero el dualismo falla por una razón fundamental. Contra lo que piensan los dualistas, el ser organismos físicos no es una característica accidental de los seres humanos, algo que ellos puedan tener o no tener. En sentido estricto, los seres humanos no tienen cuerpos, son cuerpos. La experiencia cotidiana demuestra esto: cuando a uno lo golpean, uno no pregunta ¿por qué le pegaste a mi cuerpo? Sino más bien, ¿ por qué me pegaste?

Además de esta apelación a la experiencia, hay un argumento filosófico para rechazar el dualismo. Este argumento consiste en notar que, en el caso del hombre, el sujeto de los actos intelectuales es el mismo sujeto que siente (que ha de ser un viviente corpóreo). Cuando alguien afirma, por ejemplo, «esto es un mamífero», el predicado «mamífero» designa un concepto universal aprehendido por la inteligencia, mientras que el sujeto "esto", se refiere a un individuo particular conocido por la percepción sensible. Ahora bien, quien conoce el sujeto y el predicado de un juicio unitario es siempre el mismo ser, que, en este caso, será un animal racional: un ser que siente en virtud de su animalidad, y entiende en virtud de su racionalidad ${ }^{(7)}$.

Por consiguiente, un cuerpo $\mathrm{u}$ organismo humano vivo es una persona, y la persona continúa existiendo hasta la muerte del organismo humano.

Con todo, todavía cabe que alguien conceda que los individuos de la especie humana existen hasta que dejan de ser organismos, pero que objete que sólo son personas durante ciertas porciones del tiempo de su existencia, a saber, mientras gozan del ejercicio actual de las facultades intelectuales. Sin embargo, esto también pugnaría con un hecho de experiencia: todos reconocemos que los individuos humanos que están dormidos y los que padecen un coma reversible no pierden su condición de personas. ¿Por qué? Sencillamente porque nos damos cuenta de que lo que hace que un ser sea valioso por sí mismo no es una característica accidental que ese ser tenga, sino precisamente el tipo de ser que ese individuo es ${ }^{(8)}$.

Las dos normas morales específicas más importantes que se pueden concluir del principio del respeto por el valor de la vida humana son las normas que prohíben el aborto y la eutanasia. Si lo que hemos dicho hasta ahora sobre el fundamento de la dignidad y sobre el valor de la vida es correcto, entonces el aborto procurado se revela como una injusticia gravísima: una discriminación en razón de edad, un homicidio contra los más débiles y necesitados de protección. También la eutanasia directa aparece como un atentado contra la dignidad de la persona y, además de eso, como una práctica que socava las disposiciones mismas que se requieren en los profesionales de la salud.

La práctica de la medicina no puede prosperar a menos que los doctores estén dispuestos de tal modo que inspiren confianza en sus pacientes, muchos de los cuales son extremadamente vulnerables. Los doctores no inspirarán confianza a menos que los pacientes sepan que por ninguna razón están dispuestos a matarlos. La legalización de la eutanasia socava el ímpetu por desarrollar una aproximación verdaderamente compasiva al cuidado del sufriente y del moribundo. La expresión propia de la compasión es el cuidado motivado por un sentido más o menos fuerte de solidaridad con la aflicción de la persona que sufre. Pero uno no puede cuidar de las personas matándolas ${ }^{(8)}$.

Tercer principio: se debe procurar la seguridad del paciente

Vinculado con el respeto por la vida humana, y en el contexto de la atención de enfermería, surge el principio de seguridad del paciente. Este principio exige la utilización de procesos, instrumentos y metodologías basados en evidencia científica, cuya finalidad sea coordinar esfuerzos para disminuir el riesgo de sufrir un daño prevenible en el proceso de atención de salud o de atenuar sus consecuencias.

Este ideal de seguridad, que, en su naturaleza, representa una variante de la regla primum non nocere, no es más que un aspecto de la tarea de cuidado. Lo que sucede es que el acto de cuidar y la seguridad de los pacientes son realidades que se funden en la decisión de enfermería. De este modo, el cuidado profesional y la seguridad se relacionan estrechamente con la vulnerabilidad y la confianza de las personas, y ambas actividades se despliegan a través de acciones que se dirigen a proteger, mantener y conservar la vida de un modo integral ${ }^{(9)}$.

En tal manera la seguridad del paciente se ha vuelto un principio de la atención de salud, que el MINSAL-Chile ha dispuesto que la seguridad del paciente y la calidad de la atención, son componentes fundamentales de la atención de salud, siendo necesario coordinar los esfuerzos realizados en los establecimientos de la red asistencial pública de salud en relación al cumplimiento de los estándares que se han instaurado a su efecto.

Ahora bien, la calidad del cuidado enfermero depende de la calidad y la seguridad de la asistencia al paciente. Crear un ambiente de atención propicio para la recuperación del enfermo es un aspecto fundamental de la tarea de las profesiones de la salud, sobre todo de la Enfermería. En este esfuerzo por mejorar las condiciones en que se lleva a cabo el cuidado enfermero, los indicadores de calidad tienen un papel fundamental: nos permiten objetivar situaciones relacionadas con la seguridad clínica, intentando reducir los riesgos a los que se expone el paciente que interacciona con el sistema sanitario $^{(10)}$

\section{Cuarto principio: la excelencia en el cuidado enfermero} exige una conjunción de prudencia y técnica

Uno de los tantos aportes que hizo Aristóteles a la historia del pensamiento consiste en haber distinguido con claridad entre el reino de la práxis y el reino de la póiesis, esto es, entre la ética y la técnica. Ambos saberes —notó Aristótelescoinciden en que pertenecen al ámbito de la razón práctica, o sea, en que, junto con ser conocimientos, son también directrices de la obra que ha de ser ejecutada. Sin embargo, el Estagirita vio que ellos también difieren de modo importante. La diferencia principal entre ambos órdenes de saberes radica en el fin al que cada uno se dirige: la ética tiene como fin 
propio el bonum operantis, es decir, el bien del sujeto que obra, mientras que la técnica tiene como fin propio el bonum operis, es decir, el bien de la obra producida. Veámoslo con un poco más de detalle.

La ética, mediante la acción de la prudencia y las virtudes morales, dirige la acción humana para alcanzar la perfección del agente. Por eso se dice que la ética ordena las acciones al fin común de toda la vida humana. Este fin último de la vida humana es la felicidad, que consiste en la perfección del hombre en cuanto tal. De esto se sigue que la ética se preocupa fundamentalmente por el carácter inmanente de las acciones, esto es, por el modo en que ellas modifican al propio agente que las realiza. Desde el punto de vista ético, el sujeto que obra se cualifica por las acciones que ejecuta, pues modela su personalidad moral a través de ellas. Los actos humanos tienen, así, un carácter autoconstitutivo, lo que significa que el sujeto se hace una buena o una mala persona según sus actos.

En la técnica, en cambio, lo esencial es que el producto, instrumento o procedimiento sea apto para cumplir su función. Así, se dice que un cuchillo es técnicamente bueno cuando su filo permite cortar con facilidad, su hoja es resistente y su mango es cómodo; y se dice que un procedimiento de curación es técnicamente bueno cuando restaura pronta y eficazmente la salud del órgano dañado. Lo distintivo de la técnica radica, entonces, en que orienta las acciones del hombre a conseguir un cierto fin particular.

Ésta es la razón de que, desde el punto de vista técnico, la palabra bueno tenga un sentido relativo, que nos permite hablar, por ejemplo, de un buen ladrón. Buen ladrón es, de esta manera, quien lleva a cabo de modo óptimo la acción de robar, y logra alcanzar el fin particular de apoderarse de la propiedad ajena. Desde el punto de vista ético, por el contrario, no cabe hablar de un buen ladrón, porque en ética la palabra bueno está tomada en su sentido más amplio: bueno o buena es simplemente quien es buena persona. Por otro lado, la técnica pone el énfasis en el aspecto transitivo de las acciones, o sea, en el modo como ellas modifican una materia exterior para producir un objeto o resultado externo. La producción exterior, desde luego, también tiene un aspecto inmanente, pero no es eso lo relevante para la técnica, salvo en lo que se refiere al aumento de perfección del propio hábito técnico.

Puesto que los actos de cuidado presentan, a la vez, una faz técnica y una faz ética, también en estas materias es necesario analizar las implicaciones artísticas y prudenciales de la gestión de enfermería. A este respecto, lo primero que se debe tener en cuenta es que el acto de enfermería, como acto de cuidado, incluye tanto aspectos psicosociales como aspectos profesionales y técnicos. Incluye, además, el reconocimiento de valores intrínsecos al ser humano, como el respeto a la vida o la dignidad humana, así como un esfuerzo del profesional por hacer de su trabajo una vía para el logro de la excelencia moral.

\section{CONSIDERACIONES FINALES}

La Enfermería debe estar guiada por una ética de máximos, no de mínimos. En una actividad como ésta, centrada en la persona, no basta, como dice Cortina, el cumplimiento del ethos burocrático, propio del profesional que se atiene al mínimo. Luego, no es suficiente que los enfermeros y enfermeras sean profesionales competentes, en el sentido de que aspiran al puro cumplimiento de sus deberes profesionales y legales. Ahora bien, por supuesto que es necesario los enfermeros y enfermeras cumplan sus tareas y obligaciones como miembros del equipo médico, pero el mero cumplimiento de la obligación no los convierte en profesionales excelentes, que es el estándar que debiera guiar su actividad. Lo que hace que sus decisiones alcancen la excelencia es la búsqueda del bien.

Al igual que en los otros ámbitos de la praxis, la búsqueda del bien en la salud tiene la dificultad de que se trata de una tarea circunstanciada. Los principios técnicos y éticos que puedan desarrollarse como guías de la actividad enfermera nunca se pueden aplicar axiomáticamente ante una patología o problema físico. Siempre será necesario considerar la subjetividad del enfermo. Por esto el cuidado es una tarea profesional que sólo se puede ejercer desde la singularidad del otro.

Con este trabajo hemos intentado destacar la naturaleza moral del acto de cuidado, más allá de su puro carácter técnico o artístico. A pesar de que no existen pautas para que los enfermeros realicen su trabajo adecuadamente, ya que el cuidado es siempre un fenómeno circunstanciado, sí hay principios que aseguran que el profesional de la salud no ejecute un cuidado reprobable desde el punto de vista moral. Por ejemplo, aunque no existan reglas detalladas acerca de la manera en que se debe aplicar o decidir un tratamiento paliativo, sí se sabe que nunca es lícito procurar la muerte del paciente como medio para suprimir el dolor. El reconocimiento de estos límites a la actuación profesional del equipo médico es relevante para el progreso del bienestar social. Las profesiones son siempre actividades que aspiran a contribuir al desarrollo humano. No hay verdadera profesión donde se pone en juego la dignidad de la persona.

\section{REFERENCIAS}

1. Watson J. Le caring. Philosophie et sciences de soins infirmiers. París: Seli Arslam; 1998.

2. Soledad Rivera M. Formas de conocer en Enfermería: el sustento teórico de la práctica profesional. Horiz Enferm. 2003;14(1):21-32.

3. Del Valle García S. Enfermería: el arte del cuidado. San
Juan: Universidad Nacional de Cuyo; 2010.

4. Besio RM. El acto médico: ¿una creación original? Reflexiones sobre su esencia, surgimiento y riesgos a los que se expone. Acta Bioeth. 2010;16(1):51-60.

5. Tobo N. La tecnología y el cuidado de enfermería. Pinto $\mathrm{N}$, coordinadora. Cuidado y práctica de enfermería. 
Bogotá-Medellín: Universidad Nacional de Colombia; 2000.

6. Lee P. Personhood, Dignity, Suicide, and Euthanasia. Natl Cathol Bioeth Q [Internet]. 2001 [cited 2013 July 25];1(3):329-43. Available from: http://www.ncbi.nlm. nih.gov/pubmed/12866523

7. Lee P, George RP. Body-Self Dualism in Contemporary Ethics and Politics. New York: Cambridge University Press; 2007.

8. Gormally L. Euthanasia and Assisted Suicide: Seven Reasons Why They Should Not be Legalized (1997) [Internet].
London: The Linacre Centre; 1997 [citado 2013 septiembre 05]. Disponible em: http://www.linacre.org/newsle $\sim 1 . h t m$

9. Milos P, Larraín AI. La vinculación ético-jurídica entre la gestión del cuidado y la gestión de riesgos en el contexto de la seguridad del paciente. Aquicha. 2013.

10. Ortells Abuye N, Paguina Marcos M. Indicadores de calidad y seguridad del paciente en la enfermería de urgencias: un valor seguro. Enferm Glob [Internet]. 2012 [citado 2013 septiembre 05];11(2):184-90. Disponible en: http://revistas. um.es/eglobal/article/view/eglobal.11.2.132851/133471 